NORDITA-1999/65 HE

NBI-HE-99-40

HUTP-99/054

LPTHE-99-34

hep-th/9910115

\title{
Eisenstein Series in String Theory ${ }^{\star}$
}

\author{
Niels A. Obers $\dagger$ and Boris Pioline $\ddagger^{*}$ \\ $\dagger$ Nordita and NBI, Blegdamsvej 17, DK-2100 Copenhagen, Denmark \\ obers@nordita.dk \\ $\ddagger$ Jefferson Physical Laboratory, Harvard University, Cambridge MA 02138, USA \\ pioline@ulam.harvard.edu
}

\begin{abstract}
We discuss the relevance of Eisenstein series for representing certain $G(\mathbb{Z})$-invariant string theory amplitudes which receive corrections from BPS states only. The Eisenstein series are constructed using $G(\mathbb{Z})$-invariant mass formulae and are manifestly invariant modular functions on the symmetric space $K \backslash G(\mathbb{R})$ of noncompact type, with $K$ the maximal compact subgroup of $G(\mathbb{R})$. In particular, we show how Eisenstein series of the T-duality group $S O(d, d, \mathbb{Z})$ can be used to represent one- and $g$-loop amplitudes in compactified string theory. We also obtain their non-perturbative extensions in terms of the Eisenstein series of the U-duality group $E_{d+1(d+1)}(\mathbb{Z})$.
\end{abstract}

PACS numbers: 11.25.-w;11.30.-j;2.20.Rt;2.30.Px

* Talk presented by N.O. at Strings '99, Potsdam, Germany (July 19-24, 1999). Work supported in part by TMR networks ERBFMRXCT96-0045 and ERBFMRXCT96-0090.

* On leave of absence from LPTHE, Université Pierre et Marie Curie, PARIS VI and Université Denis Diderot, PARIS VII, Boîte 126, Tour 16, $1^{\text {er }}$ étage, 4 place Jussieu, F-75252 Paris CEDEX 05, FRANCE 


\section{Introduction}

Worldsheet modular invariance has played a major role in the construction of consistent anomaly-free perturbative string models. At the one-loop level, it demands that the string partition function be invariant under the $S l(2, \mathbb{Z})$ fractional linear transformations of the modular parameter of world-sheet torus. The advent of target-space and nonperturbative dualities has brought into play yet another branch of the mathematics of automorphic forms invariant under infinite discrete groups $G(\mathbb{Z})$. These dualities arise in models with many supersymmetries, where the scalar fields take values in a rigid symmetric space $K \backslash G(\mathbb{R})$, where $K$ is the maximal compact subgroup of $G$. Duality symmetries identify points in $K \backslash G(\mathbb{R})$ differing by the right action of an infinite discrete subgroup $G(\mathbb{Z})$ of $G(\mathbb{R})$, and all physical amplitudes are constrained to be invariant under the transformations in $G(\mathbb{Z})$. This includes in particular the mapping class group $S l(d, \mathbb{Z})$ in the case of toroidal compactifications of diffeomorphism-invariant theories, the T-duality group $S O(d, d, \mathbb{Z})$ in toroidal compactifications of string theories, as well as the non-perturbative U-duality group $E_{d+1(d+1)}(\mathbb{Z})$ in maximally supersymmetric compactified M-theory or non-perturbative type II string theory [1, 2, 3] (see for instance [4, 5] for reviews and exhaustive list of references). These duality symmetries are to be contrasted with dualities between distinct string theories, which, although less tied to supersymmetry, yield much weaker constraints.

The invariance under duality symmetries, although valid for all physical amplitudes, is usually not sufficient to completely determine them. For a certain class of "BPS saturated" amplitudes however, supersymmetry gives further constraints, in the form of second order differential equations in the most favorable half-BPS saturated case [6, 0, 8]. Combining these constraints with duality invariance and behavior at the boundary of the moduli space allows in many cases to determine exact non-perturbative results not obtainable otherwise, which can then be analyzed at weak coupling to provide interesting insights on instanton calculus in string theory团 13, 14, 15 (see also Ref. 16] for lecture notes). As an illustration, Table 1 lists for a number of compactifications the (non-)perturbative symmetries that apply to all amplitudes along with the amount of supersymmetry of the theory and a selection of 1/2 and 1/4-BPS amplitudes.

The prototypical example of such an exactly computable coupling is the $R^{4}$ coupling in ten dimensional type IIB theory. This was conjectured [13] and later proved [17, 6, 8] to be a certain (non-holomorphic) automorphic form of weight 0 of the S-duality group $S l(2, \mathbb{Z})$, namely the Eisenstein series of order $3 / 2$

$$
\begin{aligned}
f_{R^{4}}^{I I B} & =\frac{1}{l_{P}^{2}} \mathcal{E}_{\mathbf{2} ; s=3 / 2}^{S l(2, \mathbb{Z})}, \\
\mathcal{E}_{\mathbf{2} ; s}^{S l(2, \mathbb{Z})} & =\sum_{(m, n) \neq 0}\left[\frac{\tau_{2}}{|m+n \tau|^{2}}\right]^{s}=\zeta(2 s) \sum_{(p, q)=1} \frac{\tau_{2}^{S}}{|p+q \tau|^{2}},
\end{aligned}
$$

‡ Exact amplitudes can also be obtained from string dualities, and yield valuable insights too, see for instance [9, 10, 11, 12. 


\begin{tabular}{|c|c|c|c|c|}
\hline theory & symmetry & SUSY & $1 / 2$ BPS & $1 / 4$ BPS \\
\hline IIB & $S l(2, \mathbb{Z})$ & 32 & $R^{4}, \lambda^{16}$ & $R^{6}, \lambda^{24}$ \\
\hline $\mathrm{M} / T^{d+1}\left(\sim \mathrm{II} / T^{d}\right)$ & $E_{d+1(d+1)}(\mathbb{Z})$ & 32 & $R^{4}, \lambda^{16}$ & $R^{6}, \lambda^{24}$ \\
\hline Het $/ T^{n}(n \leq 5)$ & $S O(n, 16+n, \mathbb{Z})$ & 16 & $R^{2}, F^{4}, \lambda^{8}$ & $F^{6}, \lambda^{12}$ \\
\hline Het $/ T^{6}$ & $S O(6,22, \mathbb{Z}) \times S l(2, \mathbb{Z})$ & 16 & $R^{2}, F^{4}, \lambda^{8}$ & $F^{6}, \lambda^{12}$ \\
\hline Het $/ T^{7}$ & $S O(8,24, \mathbb{Z})$ & 16 & $R^{2}, F^{4}, \lambda^{8}$ & $F^{6}, \lambda^{12}$ \\
\hline $\mathrm{IIB} / K_{3}$ & $S O(5,21, \mathbb{Z})$ & 16 & $R^{2}, H^{4}, \lambda^{8}$ & $H^{6}, \lambda^{12}$ \\
\hline
\end{tabular}

Table 1. (Non-)perturbative symmetries of various compactified string theories.

where $\tau=a+i / g_{s}$ is the complexified string coupling, parametrizing the upperhalf-plane $U(1) \backslash S l(2, \mathbb{R})$, and $l_{P}$ the ten-dimensional S-duality invariant Planck length $l_{P}=g_{s}^{1 / 4} l_{s}$. By expanding this series at weak coupling $\tau_{2} \rightarrow \infty$, one recovers the known tree-level and one-loop terms, along with an infinite series of exponentially suppressed terms of order $e^{-N / g_{s}}$, attributed to $N$ D-instanton configurations. It is easy to check that the above Eisenstein series is an eigenmode of the Laplacian on the upper half plane,

$\Delta_{U(1) \backslash S l(2)} \mathcal{E}_{\mathbf{2} ; s}^{S l(2, \mathbb{Z})}=\frac{s(s-1)}{2} \mathcal{E}_{\mathbf{2} ; s}^{S l(2, \mathbb{Z})}, \quad \Delta_{U(1) \backslash S l(2)}=\frac{1}{2} \tau_{2}^{2}\left(\partial_{\tau_{1}}^{2}+\partial_{\tau_{2}}^{2}\right)$.

Supersymmetry on the other hand requires the exact $R^{4}$ coupling to be an eigenmode of the same Laplacian with the precise eigenvalue $3 / 8$, which together with the assumption of at most polynomial growth at weak coupling uniquely selects the Eisenstein series of order $3 / 2$.

In this talk we discuss the construction and utility of automorphic forms of the duality groups $G(\mathbb{Z})$ generalizing the Eisenstein series of $S l(2, \mathbb{Z})$ (see Ref. [15] for an extensive treatment). For the representation theory of these groups, in particular the multiplets of half-BPS states that we will use, we refer the reader to the review [5] and its very brief summary [18. Section 2 presents the general construction of Eisenstein series, Section 3 its applications to perturbative string amplitudes; Section 4 extends these results to the full non-perturbative expression and finally Section 5 mentions some open directions.

\section{Generalized Eisenstein series}

We focus on string or M-theory models whose moduli space is spanned by a symmetric space of non-compact type

$$
K \backslash G(\mathbb{R}) \quad, \quad G(\mathbb{R})=K \cdot A \cdot N,
$$

where $K$ is the maximal compact subgroup of $G(\mathbb{R})$ and we have also indicated the Iwasawa decomposition of $G(\mathbb{R})$ into elements of the maximal compact, Abelian and nilpotent subgroups $K, A, N$ respectively. This structure may describe only a part of 
the moduli space, like the internal metric for toroidal compactifications or the NeveuSchwarz moduli for perturbative strings, or the full moduli space when protected by supersymmetry. Duality symmetries identify different points in this moduli space and form an infinite discrete group $G(\mathbb{Z})$ of the classical symmetry group $G(\mathbb{R})$, acting from the right on the coset $K \backslash G(\mathbb{R})$. The BPS states are labelled by a set of integer charges $m$ and fall into certain representations $\mathcal{R}$ of the duality group. The mass of half-BPS states is given by the manifestly $G(\mathbb{Z})$-invariant form

$$
\mathcal{M}^{2}(\mathcal{R})=m \cdot M_{\mathcal{R}}(g) \cdot m .
$$

Here, the moduli matrix $M_{\mathcal{R}}$ in representation $\mathcal{R}$ is given by

$$
M_{\mathcal{R}}(g)=\mathcal{R}^{t} \mathcal{R}(g) \quad, \quad g \in A \cdot N,
$$

where $g$ denotes an element in the coset $K \backslash G(\mathbb{R})$. Moreover, there is in general a half-BPS constraint, quadratic in the charges, that can be written schematically as

$$
\text { 1/2-BPS: } \quad k=m \wedge m=0,
$$

where the cup product $m \wedge m$ denotes all but the highest of the irreducible components of the symmetric tensor product $\mathcal{R} \otimes_{s} \mathcal{R}$. The constraint thus amounts to requiring that $\mathcal{R} \otimes_{s} \mathcal{R}$ be an irreducible representation.

To be more specific, in the case of the $S l(n, \mathbb{Z})$ duality groups (of toroidally compactified diffeomorphism invariant theories) we will be mostly interested in the fundamental and antifundamental representations, labelled by the charges $m^{i}$ and $m_{i}$ respectively. In this case the moduli matrix $M$ in (6) is simply the (inverse) metric $g_{i j}\left(g^{i j}\right)$ on the torus. For the T-duality group of toroidally compactified string theory we will need the vector, spinor and conjugate spinor representations, while for the Uduality group of M-theory the particle, string and membrane representations will be of relevance (see [5, 18, 15] for details).

Given the duality group $G(\mathbb{Z})$, one may follow the definition of Eisenstein series given in the mathematical literature by 19

$$
\mathcal{E}_{\left\{w_{i}\right\}}^{G(\mathbb{Z})}(g)=\sum_{h \in G(\mathbb{Z}) / N} \prod_{i=1}^{r} a_{i}(g h)^{-w_{i}}
$$

where $w_{i}$ is now an arbitrary $r$-dimensional vector $(r=\operatorname{Rank} G)$ in weight space, and $a(g)$ is the Abelian component of $g$ in the Iwasawa decomposition (4). Note that this definition is manifestly $K$-invariant on the left and $G(\mathbb{Z})$-invariant on the right. This definition is however hardly tractable, and we rather define our generalized Eisenstein series [14, 15] as

$$
\mathcal{E}_{\mathcal{R} ; s}^{G(\mathbb{Z})}(g)=\sum_{m \in \Lambda_{\mathcal{R}} \backslash\{0\}} \delta(m \wedge m)\left[m \cdot \mathcal{R}^{t} \mathcal{R}(g) \cdot m\right]^{-s}
$$

for any symmetric space $K \backslash G(\mathbb{R})$, any representation $\mathcal{R}$ of $G$ and any order $s$. This definition, albeit less genera][3] than (8), has a more transparent physical meaning: the $\S$ Choosing $w$ along a highest-weight vector $\lambda_{\mathcal{R}}$ associated to a representation $\mathcal{R}$ reduces (8) to (9) where $w=s \lambda_{\mathcal{R}}$, up to an $s$-dependent factor. These two definitions generalize the two terms of the last equality in (2) to higher rank groups. 
lattice $\Lambda_{\mathcal{R}}$ labels the set of BPS states in the representation $\mathcal{R}$ of the duality group, $\mathcal{M}^{2}=m \cdot \mathcal{R}^{t} \mathcal{R}(g) \cdot m$ gives their mass squared (or tension), and the $\delta$-function imposes the half-BPS condition (7). This ensures that the sum runs over one $G(\mathbb{Z})$ orbit only, and is in fact a necessary requirement for the Eisenstein series to be an eigenmode of the Laplacian on the symmetric space. The eigenvalue follows simply from group theory,

$$
\Delta_{K \backslash G} \mathcal{E}_{\mathcal{R}_{\lambda} ; s}^{G(\mathbb{Z})}=s(\lambda, \rho-s \lambda) \mathcal{E}_{\mathcal{R}_{\lambda} ; s}^{G(\mathbb{Z})}
$$

where $\lambda$ is the highest weight of the representation and $\rho$ is the Weyl vector 15. This expression implies in particular that Eisenstein series associated to representations related by outer automorphisms, i.e. symmetries of the Dynkin diagram, are degenerate under $\Delta_{K \backslash G}$, as well as two Eisenstein series of same representation but order $s$ and the "dual" value $[(\lambda, \rho) /(\lambda, \lambda)]-s$. Up to these identifications, we expect that, aside from cusp forms, a basis of invariant functions on the space is spanned by the representations corresponding to the nodes of the Dynkin diagram.

\section{Perturbative string amplitudes}

\subsection{One-loop modular integral}

Under toroidal compactification on a torus $T^{d}$, any string theory exhibits the T-duality symmetry $S O(d, d, \mathbb{Z})$, and all amplitudes should be expressible in terms of modular forms of this group. For half-BPS saturated couplings, the one-loop amplitude often reduces to an integral of a lattice partition function over the fundamental domain $\mathcal{F}$ of the moduli space of genus-1 Riemann surfaces,

$$
I_{d}=2 \pi \int_{\mathcal{F}} \frac{d^{2} \tau}{\tau_{2}^{2}} Z_{d, d}(g, B ; \tau),
$$

where $Z_{d, d}$ is the partition function (or theta function) of the even self-dual lattice describing the toroidal compactification. This is for instance the case for $R^{4}$ couplings in type II strings on $T^{d}$, or $R^{2}$ or $F^{2}$ couplings in type II on $K_{3} \times T^{2}$.

It is natural to expect a connection between this one-loop modular integral and the $S O(d, d, \mathbb{Z})$ Eisenstein series defined above. As is well known, the $\tau$-integral can be carried out by the method of orbits, which corresponds to a large volume expansion of the integral. This yields [14, 20]

$$
I_{d}=\frac{2 \pi^{2}}{3} V_{d}+2 V_{d} \mathcal{E}_{d ; s=1}^{S l(d, \mathbb{Z})}+\ldots
$$

and exhibits the order $1 S l(d, \mathbb{Z})$ Eisenstein series in the fundamental representation. The omitted terms are exponentially suppressed world-sheet instanton terms that can be written down exactly and simplify for the low-dimensional cases, reducing to the standard results:

$$
I_{1}=\frac{2 \pi^{2}}{3}\left(R+\frac{1}{R}\right), \quad I_{2}=-2 \pi \log \left(T_{2} U_{2}|\eta(T) \eta(U)|^{4}\right) .
$$


Treating these simple cases first, it is not difficult to show that they can be rewritten as the sum of the $S O(d, d, \mathbb{Z})$ Eisenstein series of order 1 in the spinor and conjugate spinor representations:

$$
I_{d}=2 \mathcal{E}_{\mathbf{S} ; s=1}^{S O(d, d, \mathbb{Z})}+2 \mathcal{E}_{\mathbf{C} ; s=1}^{S O(d, d, \mathbb{Z})} \quad, \quad d=1,2 .
$$

In particular, the result is manifestly invariant under the extended T-duality $O(d, d, \mathbb{Z})$, where the extra generator exchanges the two spinors.

To arrive at a similar claim for $d>2$, one considers the eigenvalues of the one-loop integral under both the Laplacian $\Delta_{S O(d, d)}$ on the moduli space $S O(d) \times$ $S O(d) \backslash S O(d, d, \mathbb{R})$, as well as under another second order differential operator $\square_{d}$. This operator is given by

$$
\square_{d}=\Delta_{G l(d)}-\frac{1}{8}\left(g_{i j} \frac{\partial}{\partial g_{i j}}\right)^{2}=\Delta_{S l(d)}+\frac{2-d}{8 d}\left(g_{i j} \frac{\partial}{\partial g_{i j}}\right)^{2}
$$

which is non-invariant under $S O(d, d)$, but still invariant under complete T-duality on all directions, and happens to have $I_{d}$ as an eigenvector. The resulting eigenvalues are 15.

$$
\Delta_{S O(d, d)} I_{d}=\frac{d(2-d)}{4} I_{d} \quad, \quad \square_{d} I_{d}=\frac{d(2-d)}{8} I_{d} .
$$

On the other hand, the corresponding eigenvalues under these operators of the Eisenstein series in the vector, spinor and conjugate spinor representation are given by,

$$
\begin{aligned}
& \Delta(\mathbf{V}, s)=s(s-d+1), \quad \Delta(\mathbf{S}, s)=\Delta(\mathbf{C}, s)=\frac{s d(s-d+1)}{4} \\
& \square_{d} \mathcal{E}_{\mathbf{V} ; s}^{S O(d, d, \mathbb{Z})}=\frac{s(s-d+1)}{2} \mathcal{E}_{\mathbf{V} ; s}^{S O(d, d, \mathbb{Z})} \\
& \square_{d} \mathcal{E}_{\mathbf{S} ; s=1}^{S O(d, d, \mathbb{Z})}=\frac{d(2-d)}{8} \mathcal{E}_{\mathbf{S} ; s=1}^{S O(d, d, \mathbb{Z})} \\
& \square_{d} \mathcal{E}_{\mathbf{C} ; s=1}^{S O(d, d, \mathbb{Z})}=\frac{d(2-d)}{8} \mathcal{E}_{\mathbf{C} ; s=1}^{S O(d, d, \mathbb{Z})} .
\end{aligned}
$$

Comparing (16) with (17)-(20), we see that the candidate Eisenstein series for the one-loop integral are restricted to the order $s=1$ spinor and conjugate spinor series, together with the order $s=d / 2-1$ vector series and their duals. Another constraint comes from comparison of the large volume expansion (12) with the expansion of the Eisenstein series. These two requirements then enable one to show that the one-loop integral $I_{d}$ in (11) can be represented for $d \geq 3$ as

$$
I_{d}=2 \frac{\Gamma\left(\frac{d}{2}-1\right)}{\pi^{\frac{d}{2}-2}} \mathcal{E}_{\mathbf{V} ; s=\frac{d}{2}-1}^{S O(d, d, \mathbb{Z})}=2 \mathcal{E}_{\mathbf{S} ; s=1}^{S O(d, d, \mathbb{Z})}=2 \mathcal{E}_{\mathbf{C} ; s=1}^{S O(d, d, \mathbb{Z})} .
$$

Here, the first equality is a theorem and the last two are well-supported conjectures [15].

As a practical application of this result, let us consider the conjectured duality between the heterotic string on $T^{4}$ and type IIA on $K_{3}$. On the heterotic side, the halfBPS states are momentum and winding states, and transform as a vector of $S O(4,20, \mathbb{Z})$. On the type IIA side at the $T^{4} / \mathbb{Z}_{2}$ orbifold point, these states correspond instead to 
even branes wrapped on invariant cycles of $T^{4}$, plus "fractional" branes stuck at the fixed points of the orbifolds. Restricting to an $[S O(4) \times S O(4)] \backslash S O(4,4)$ subspace of the moduli space, it is easy to see that under duality, the vector of $S O(4,4)$ should be mapped to the conjugate spinor: the duality therefore corresponds to a triality in $S O(4,4)$, and using our last result (21), it is easy to see that $I_{4}$ is indeed invariant under heterotic-type IIA duality. This fact will be instrumental in checking the duality conjecture at the level of $F^{4}$ couplings 21 .

\subsection{Genus g modular integral}

Similar methods carry over to higher-loop amplitudes, such as the higher-genus analogue of (11), namely the integral of a lattice partition function on the $3 g$ - 3 -dimensional moduli space $\mathcal{M}_{g}$ of genus $g$ curves

$$
I_{d}^{g}=\int_{\mathcal{M}_{g}} d \mu Z_{d, d}^{g}\left(g_{i j}, B_{i j} ; \tau\right)
$$

where $Z_{d, d}^{g}$ is the genus $g$ lattice sum. In this case the modular group is $S p(g, \mathbb{Z})$ and one may derive by similar methods as in the one-loop case the eigenvalue condition,

$$
\Delta_{S O(d, d)} I_{d}^{g}=\frac{d g(g+1-d)}{4} I_{d}^{g} .
$$

Comparison with (17) shows that this eigenvalue agrees with the order $s=g$ Eisenstein series in the spinor and conjugate spinor representation. This leads us to the conjecture that the $g$-loop integral (22) is (up to an overall factor) given by the $S O(d, d, \mathbb{Z}$ ) Eisenstein series of order $g$ in the spinor representation:

$$
I_{d}^{g} \propto \mathcal{E}_{\mathbf{S} ; s=g}^{S O(d, d, \mathbb{Z})}+\mathcal{E}_{\mathbf{C} ; s=g}^{S O(d, d, \mathbb{Z})}
$$

where the superposition of the two spinor representations is required by the $O(d, d, \mathbb{Z})$ invariance of the integrand. Note that normalizing (24) would require a knowledge of the Weil-Peterson volume of the moduli space of genus $g$ curves.

Though less substantiated than the one-loop conjecture (21), Equation (24) is strongly reminiscent of the genus $g$ partition function of the $N=4$ topological string 222 on $T^{2}$ which was shown to be exactly given by the Eisenstein series of order $s=g$ in the spinor representation $\mathcal{E}_{\mathbf{2} ; s=g}^{S l(2, \mathbb{Z})}(T)$ [23]. This result was subsequently used to derive a set of higher derivative topological couplings $R^{4} H^{4 g-4}$ in type IIB string compactified over $T^{2}$ [24]. Our conjecture (24) suggests a natural generalization of these results to lower dimensions: The $R^{4} H^{4 g-4}$ couplings between 4 gravitons and $4 g-4$ Ramond three-form field-strengths in type IIA compactified on $T^{d}, d \leq 4$ are given at genus $g$ by the $S O(d, d, \mathbb{Z})$ constrained Eisenstein series in the spinor representation with insertions of $4 g-4$ charges:

$$
I=\int d^{10-d} x \sqrt{-\gamma} \sum_{m} \delta(m \wedge m) e^{6(g-1) \phi} \frac{R^{4}\left(m \cdot H_{R R}\right)^{4 g-4}}{(m \cdot M(\mathbf{S}) \cdot m)^{3 g-2}},
$$

where $\phi$ is the T-duality invariant dilaton, related to the ten-dimensional coupling as $e^{-2 \phi}=V_{d} / g_{s}^{2} l_{s}^{d}$, and we work in units of $l_{s}$. A similar conjecture also holds for 
the coupling computed by the topological B-model [22], and states that the $R^{4} F^{4 g-4}$ couplings between 4 gravitons and $4 g-4$ Ramond two-form field-strengths in type IIA compactified on $T^{d}, d \leq 6$ are given at genus $g$ by the $S O(d, d, \mathbb{Z})$ constrained Eisenstein series in the conjugate spinor representation with insertions of $4 g-4$ charges:

$$
I=\int d^{10-d} x \sqrt{-\gamma} \sum_{m} \delta(m \wedge m) e^{6(g-1) \phi} \frac{R^{4}\left(m \cdot F_{R R}\right)^{4 g-4}}{(m \cdot M(\mathbf{C}) \cdot m)^{3 g-2}} .
$$

Note that the results (25) and (26) involve covariant modular functions instead of invariant ones, but behave like Eisenstein series of order $3 g-2-(4 g-4) / 2=g$ as far as their eigenvalue and decompactification limit are concerned. They generalize the $S l(2, \mathbb{Z})$ modular functions $f^{p, q}=\hat{\sum} \tau_{2}^{(p+q) / 2} /\left[(m+n \tau)^{p}(m+n \bar{\tau})^{q}\right]$ invariant up to a phase, that were also used in the context of non-perturbative type IIB string in 25, 26.

\section{Non-perturbative string amplitudes}

\section{1. $R^{4}$ couplings in toroidally compactified type II}

While Eisenstein series provide a nice way to rewrite one-loop integrals such as (11), their utility becomes even more apparent when trying to extend the perturbative computation into a non-perturbatively exact result. Indeed, a prospective exact threshold should reduce in a weak coupling expansion to a sum of T-duality invariant Eisenstein-like perturbative terms, plus exponentially suppressed contributions, and Eisenstein series of the larger non-perturbative duality symmetry are natural candidates in that respect.

Four graviton $R^{4}$ couplings in maximally supersymmetric theories have been argued in dimension $D \geq 8$ to receive no perturbative corrections beyond the tree-level and one-loop terms, and to be eigenmodes of the Laplacian on the full scalar manifold $K \backslash E_{d+1(d+1)}(\mathbb{R})$ as a consequence of supersymmetry. We assume both properties to persist in lower dimensions as well, so that from the first property we have

$$
f_{R^{4}}=2 \zeta(3) \frac{V_{d}}{g_{s}^{2}}+I_{d}+\text { non pert. }
$$

This result can be suggestively rewritten in terms of T-duality Eisenstein series as,

$$
f_{R^{4}}=\frac{V_{d}}{g_{s}^{2}} \mathcal{E}_{\mathbf{1} ; s=3 / 2}^{S O(d, d, \mathbb{Z})}+\mathcal{E}_{\mathbf{S} ; s=1}^{S O(d, d, \mathbb{Z})}+\text { non pert. }
$$

While the Ramond scalars are decoupled from the perturbative expansion by PecceiQuinn symmetries, the full non-perturbative result should depend on all the scalars in the symmetric space $K \backslash E_{d+1(d+1)}(\mathbb{R})$. The $R^{4}$ threshold should thus be an automorphic form of $E_{d+1(d+1)}(\mathbb{Z})$ with asymptotic behavior as in (28). To obtain the nonperturbative extension of this result, we use the fact that the singlet and spinor representations of the T-duality group are unified into the string representation [27, 28, 5] of the U-duality group, the tension of which is given by

$$
\mathcal{T}^{2}=m^{2}+\frac{V_{d}}{g_{s}^{2}} \mathcal{M}^{2}(\mathbf{S})+\frac{V_{d}^{2}}{g_{s}^{4}}, \mathcal{M}^{2}(\boldsymbol{O}),
$$


where $\boldsymbol{O}$ is an extra representation that appears when $d \geq 4$. From this group theory fact, one arrives at the non-perturbative generalization of (28): The exact four-graviton $R^{4}$ coupling in toroidal compactifications of type II theory on $T^{d}$, or equivalently Mtheory on $T^{d+1}$, is given, up to a factor of Newton's constant, by the Eisenstein series of the U-duality group $E_{d+1(d+1)}(\mathbb{Z})$ in the string multiplet representation, with order $s=3 / 2$ :

$$
f_{R^{4}}=\frac{V_{d+1}}{l_{M}^{9}} \mathcal{E}_{\text {string;s=3/2 }}^{E_{d+1(d+1)}(\mathbb{Z})} .
$$

Here $l_{M}$ is the eleven-dimensional Planck length, $V_{d+1}=R_{s} V_{d}$ the volume of the Mtheory torus $T^{d+1}$. The quantity $V_{d+1} / l_{M}^{9}=l_{P}^{d-8}$ is the U-duality invariant gravitational constant in dimension $D=10-d$. As an immediate check, the proposal has the appropriate scaling dimension $d+1-9+3 \times 2$ for an $R^{4}$ coupling in dimension $D=10-d$.

Using the eigenvalue equation (10) we can in fact determine the corresponding eigenvalues of (30) under the Laplacian on the symmetric space $K \backslash E_{d+1(d+1)}(\mathbb{R})$

$$
\Delta_{E_{d+1(d+1)}} f_{R^{4}}=\frac{3(d+1)(2-d)}{2(8-d)} f_{R^{4}} .
$$

This property could in principle be proved from supersymmetry arguments along the lines of [6, 8], and holds order by order in the the weak coupling expansion. In fact, using the eigenvalues of the particle and membrane representations of the U-duality group one arrives at the conjecture that the non-perturbative $R^{4}$ amplitude in M-theory on $T^{d+1}$ has three equivalent forms,

$\frac{V_{d+1}}{l_{M}^{9}} \mathcal{E}_{\text {string } ; s=3 / 2}^{E_{d+1(d+1)}(\mathbb{Z})}=\frac{\Gamma(d / 2-1)}{\pi^{d / 2-2}} \mathcal{E}_{\text {particle } ; s=d / 2-1}^{E_{d+1(d+1)}(\mathbb{Z})}=\frac{V_{d+1}}{l_{M}^{9}} \mathcal{E}_{\text {membrane } ; s=1}^{E_{d+1(d+1)}(\mathbb{Z})}$.

Again, it is easy to check that the scaling dimensions match.

As a justification of the claim (30), it can be shown that it reproduces the perturbative contributions in (27) in a weak coupling expansion. Moreover, taking the $d=4$ case as an example the non-perturbative terms can be interpreted as superposition of Euclidean D0 and D2-branes wrapped on a one-cycle or a threecycle of $T^{4}$. In addition to these terms, there are further contributions which behave superficially as $e^{-1 / g_{s}^{2}}$. Such non-perturbative effects are certainly unexpected in toroidal compactifications to $D>4$, since there are no half-BPS instanton configurations with this action (the NS5-brane does have a tension scaling as $1 / g_{s}^{2}$, but it can only give rise to Euclidean configurations with finite actions when $D \leq 4$ ). The matching of the tree-level and one-loop contributions together with the consistent interpretation of the D-brane contribution is however a strong support to our conjecture.

\section{2. $R^{4} H^{4 g-4}$ and $R^{4} F^{4 g-4}$ couplings}

Using the manifestly T-duality invariant forms (25) and (26) of the $g$-loop amplitudes, it is also straightforward to propose a non-perturbative completion of the $R^{4} H^{4 g-4}$ and $R^{4} F^{4 g-4}$ couplings, invariant under the full U-duality group. For that purpose, we note that the set of three-form field-strengths in M-theory compactified on $T^{d+1}$ fall 
into a representation of $E_{d+1(d+1)}$ dual to the string multiplet. The string multiplet decomposes under $S O\left(d, d, \mathbb{Z}\right.$ ) into a singlet (the Neveu-Schwarz $H_{N S}$ ), a spinor (the Ramond three-forms obtained by reducing the M-theory four-form field-strength), as well as further terms for $d \geq 4$. We therefore conjecture that the $R^{4} H^{4 g-4}$ couplings between 4 gravitons and $4 g-4$ three-form field-strengths in M-theory compactified on $T^{d+1}, d \leq 4$ are exactly given, up to a power of Newton's constant, by the $E_{d+1(d+1)}(\mathbb{Z})$ constrained Eisenstein series in the string representation with insertions of $4 g-4$ charges:

$$
I=\frac{V_{d+1}}{l_{M}^{9}} \int d^{10-d} x \sqrt{-\gamma} \sum_{m} \delta(m \wedge m) \frac{R^{4}(m \cdot H)^{4 g-4}}{(m \cdot M(\text { string }) \cdot m)^{3 g-\frac{3}{2}}} .
$$

As an immediate check, we note that this proposal has the appropriate scaling dimension, while the expression also reproduces the tree-level interaction involving the Neveu-Schwarz three-form only. Moreover, the result (33) reproduces the $g$-loop result (25). The analysis of non-perturbative effects is as in the $R^{4}$ case, and shows order $e^{-1 / g_{s}}$ D-brane effects as well as, for $d \geq 4$, contributions superficially of order $e^{-1 / g_{s}^{2}}$.

Similarly, for the case of non-perturbative $R^{4} F^{4 g-4}$ couplings, we note that the two-form field-strengths of M-theory compactified on $T^{d+1}$ transform as the dual of the particle multiplet. This makes it natural to propose that

$$
I=\int d^{10-d} x \sqrt{-\gamma} \sum_{m} \delta(m \wedge m) \frac{R^{4}(m \cdot F)^{4 g-4}}{(m \cdot M(\text { particle }) \cdot m)^{4 g-5+\frac{d}{2}}},
$$

where the power $4 g-5+d / 2$ has been set by dimensional analysis. The particle multiplet decomposes as a vector and conjugate spinor of $S O(d, d, \mathbb{Z})$ in that order, so that this proposal implies a one-loop term given by the $S O(d, d, \mathbb{Z})$ Eisenstein series of order $2 g-3+d / 2$ in the vector representation, plus a higher perturbative term which should reproduce the genus $g$ term (26). Due to the presence of constraints, this statement has not been proved at present.

\section{Open directions}

The analysis presented here has focussed on half-BPS saturated couplings in theories with maximal supersymmetry. It would be interesting to extend our techniques to (i) couplings preserving a lesser amount of supersymmetry, and (ii) half BPS states in theories with less supersymmetry. Given that the quadratic half-BPS constraint imposes second order differential equations and that the quarter-BPS condition is cubic in the charges, one may envisage that quarter-BPS saturated couplings should be eigenmodes of a cubic Casimir operator, and expressable as generalized Eisenstein series with quarter-BPS conditions inserted.

On a more mathematical level, our results provide a wealth of explicit examples of modular functions on symmetric spaces of non-compact type $K \backslash G$, with $G$ a real simply laced Lie group in the normal real form, that generalize the Eisenstein series on the fundamental domain of the upper half-plane. We have not addressed the question of the analyticity of Eisenstein series with respect to the order $s$. Unfortunately, the presence 
of constraints tends to give rise to ill-behaved expansions, which is the mathematical counterpart of the physical problem raised above of understanding the instanton effects superficially of order $e^{-1 / g_{s}^{2}}$.

It would also be interesting to understand more precisely what Eisenstein series are needed to generate the spectrum of the Laplace operator for any eigenvalue (note in that respect that the order $s$ is no longer a good parametrization, since the relation between the eigenvalue and $s$ depends on the representation). From the point of view of harmonic analysis however, Eisenstein series are the least interesting part of the spectrum on such manifolds, which should also include a discrete series of cusp forms. Hopefully string theory will provide an explicit example of these elusive objects.

\section{References}

[1] C.M. Hull and P.K. Townsend, Nucl. Phys. B451 (1995) 525, hep-th/9505073.

[2] P.K. Townsend, Phys. Lett. B350 (1995) 184, hep-th/9501068.

[3] E. Witten, Nucl. Phys. B443 (1995) 85, hep-th/9503124.

[4] A. Giveon, M. Porrati and E. Rabinovici, Phys. Rept. 244 (1994) 77, hep-th/9401139.

[5] N.A. Obers and B. Pioline, Phys. Rept. 318 (1999) 113, hep-th/9809039.

[6] B. Pioline, Phys. Lett. B431 (1998) 73, hep-th/9804023.

[7] S. Paban, S. Sethi and M. Stern, J. High Energy Phys. 06 (1998) 012, hep-th/9806028.

[8] M.B. Green and S. Sethi, Phys. Rev. D59 (1999) 046006, hep-th/9808061.

[9] J.A. Harvey and G. Moore, Phys. Rev. D57 (1998) 2323, hep-th/9610237.

[10] C. Bachas et al., Nucl. Phys. B509 (1998) 33, hep-th/9707126.

[11] A. Gregori et al., Nucl. Phys. B510 (1998) 423, hep-th/9708062.

[12] I. Antoniadis, B. Pioline and T.R. Taylor, Nucl. Phys. B512 (1998) 61, hep-th/9707222.

[13] M.B. Green and M. Gutperle, Nucl. Phys. B498 (1997) 195, hep-th/9701093.

[14] E. Kiritsis and B. Pioline, Nucl. Phys. B508 (1997) 509, hep-th/9707018.

[15] N.A. Obers and B. Pioline, (1999), hep-th/9903113.

[16] E. Kiritsis, Lectures given at ICTP Trieste Spring Workshop on Superstrings and Related Matters, Trieste, 1999, hep-th/9906018.

[17] N. Berkovits, Nucl. Phys. B514 (1998) 191, hep-th/9709116.

[18] N.A. Obers and B. Pioline, 2nd Conference on Quantum Aspects of Gauge Theories, Supersymmetry and Unification, Corfu, 1998, hep-th/9812139.

[19] Harish-Chandra, Automorphic Forms on Semisimple Lie Groups, Lecture Notes in Mathematics No. 62 (Springer Verlag, 1968).

[20] E. Kiritsis and N.A. Obers, J. High Energy Phys. 10 (1997) 004, hep-th/9709058.

[21] E. Kiritsis, N.A. Obers and B. Pioline, J. High Energy Phys. 01 (2000) 029, hep-th/0001083.

[22] N. Berkovits and C. Vafa, Nucl. Phys. B433 (1995) 123, hep-th/940719d.

[23] H. Ooguri and C. Vafa, Nucl. Phys. B451 (1995) 121, hep-th/9505183.

[24] N. Berkovits and C. Vafa, Nucl. Phys. B533 (1998) 181, hep-th/9803145.

[25] A. Kehagias and H. Partouche, Phys. Lett. B422 (1998) 109, hep-th/9710023.

[26] M.B. Green, M. Gutperle and H. Kwon, Phys. Lett. B421 (1998) 149, hep-th/9710151.

[27] S. Elitzur et al., Nucl. Phys. B509 (1998) 122, hep-th/9707217.

[28] N.A. Obers, B. Pioline and E. Rabinovici, Nucl. Phys. B525 (1998) 163, hep-th/9712084. 